\title{
Leishmania amazonensis sabotages host cell SUMOylation for intracellular
} survival

\author{
Kendi Okuda ${ }^{1}$, Miriam Maria Silva Costa Franco', Ari Yasunaga ${ }^{2}$, Ricardo

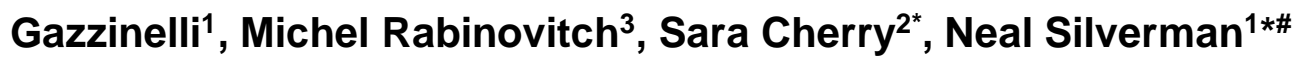

1. Division of Infectious Diseases and Immunology, Department of Medicine, University of Massachusetts Medical School, Worcester, USA.

2. Department of Microbiology, University of Pennsylvania, Philadelphia, Pennsylvania, USA

3. Department of Microbiology, Immunology and Parasitology, Escola Paulista de Medicina, Universidade Federal de São Paulo, São Paulo, Brazil

${ }^{*}$ Co-corresponding authors \# Lead Contact

Keywords: Leishmania, macrophages, Drosophila, hemocytes, high-throughput screening, SUMO, parasitophorous vacuole, SUMOylation.

\begin{abstract}
Leishmania parasites use elaborate virulence mechanisms to invade and thrive in macrophages. These virulence mechanisms inhibit host cell defense responses and generate a specialized replicative niche, the parasitophorous vacuole. In this work, we performed a genome-wide RNAi screen in Drosophila macrophage-like cells to identify host factors necessary for Leishmania amazonensis infection. This screen identified 52 conserved genes required specifically for parasite entry, including several components of the SUMOylation machinery. Further studies in mammalian macrophages found that L. amazonensis infection inhibited SUMOylation within infected macrophages and this inhibition enhanced parasitophorous vacuole growth and parasite proliferation through modulation of multiple genes especially ATP6VOD2, which in turn effects CD36 expression and cholesterol levels. Together, these data suggest that parasites actively sabotage host SUMOylation and alter host transcription to improve their intracellular niche and enhance their replication.
\end{abstract}




\section{Introduction}

Leishmania parasites use multiple strategies to thrive in mammalian phagocytes. One striking example is the use of virulence factors to evade or down regulate the host immune response. For example, amastigote forms of the parasite invade macrophages, and express surface molecules that engage phagocytic receptors that promote 'silent' or anti-inflammatory cell invasion, typically observed with the engulfment of apoptotic cells [1-5]. Inside macrophages, amastigotes live within specialized replicative organelles known as parasitophorous vacuoles (PVs), which are modified mature phagosomes. Amastigotes create a hospitable niche in the PV through secretion of virulence factors that dampen host cell defenses. These secreted factors work through many mechanisms including altering host protein expression and modulating posttranslational protein modifications [6,7, reviewed in 8,9-13]. Our understanding of the virulence mechanisms used by these parasites is incomplete while a deeper understanding is essential to develop new strategies to combat leishmaniasis, which threatens 0.9 million people annually [14].

Mammalian models of Leishmania infection have been essential to build our current understanding of leishmaniasis. However, other models of infection, such as Drosophila melanogaster, are attractive alternatives because they share many key signaling pathways with mammals and have powerful genetic and genomic techniques readily accessible. Here, we exploited the Drosophila SL2 macrophage-like cell line in a genome-wide RNAi screen to identify host factors required for $L$. amazonensis infection. With this screen, we identified and validated 52 conserved host factors specifically involved in L. amazonensis entry but not Escherichia colinor Staphylococcus aureus phagocytosis. These hits were particularly enriched in SUMOylation factors.

SUMOylation is a ubiquitous post-translational modification in which a Small Ubiquitin-like MOdifier protein (Sumo) is conjugated to lysine (Lys) residues on target proteins, through the sequential action of E1 activating (Sae1/Uba2 dimer), E2 conjugating (Ubc9) and E3 ligases (several of them) enzymes [15]. SUMOylation regulates gene expression, protein function, localization or activity [16], and 6,747 different SUMOylated proteins were cataloged in HeLa and U2OS cell lines [17]. 
Interestingly, many pathogens, including virus, bacteria and protozoans, target SUMOylation to exploit host cellular functions or to inhibit cell defense responses [1823]. Here, we observed that intracellular L. amazonensis amastigotes efficiently inhibited overall macrophage SUMOylation, reducing this post-translational modification to nearly undetectable levels. Moreover, SUMO1 or SUMO2 depleted cells presented enhanced parasite growth with larger PVs, higher expression of ATP6V0D2, and consequently elevated $C D 36$ and cholesterol levels. Therefore, our data suggest that inhibition of SUMOylation is a novel virulence mechanism of $L$. amazonensis parasites, used for successful infection of macrophages.

\section{Results}

\section{The Drosophila cell model of $L$. amazonensis infection}

Drosophila macrophages, also known as plasmatocytes, play key roles in immune defense and development [24]. Previously, we demonstrated that these macrophages use CD36-like scavenger receptors to phagocytose and control proliferation of $L$. amazonensis in the hemolymph of adult flies [25]. In this work, we determined if the Drosophila macrophage-like SL2 cell line would phagocytose parasites, in particular the amastigote form that is responsible for repeated cycles of macrophage infections in mammalian hosts. SL2 cells were infected with antibody free $L$. amazonensis amastigotes which were promptly phagocytosed and observed within SL2 cells (Fig 1A). By $48 \mathrm{~h}$ post infection, parasites were inside small acidic (Lysotracker positive) vacuoles (Fig 1B), and by $96 \mathrm{~h}$ post infection intracellular parasites were mostly cleared from these cells (not shown).

As PV biogenesis and parasite proliferation were not observed in the SL2 cell model, this allowed us to specifically focus on the phagocytosis and entry of amastigotes, a step that is essential for leishmaniasis development. To quantify intracellular parasites, SL2 cells were infected with GFP-expressing amastigotes, fixed and extracellular parasites were immunostained with anti-Leishmania serum. The ratio of intracellular parasites (immunostaining negative) and SL2 cell number (Hoechst 33342 stain of nucleus) was scored microscopically using image analysis software (Figure S1A, B). The phagocytosis of parasites increased over the $4 \mathrm{~h}$ time course of this assay, with 
$\sim 70 \%$ of the internalization completed by $90 \mathrm{~min}$ (Fig S1C). The effect of several multiplicities of infection was analyzed at 90 min post infection (pi). While the total number of internalized parasites increased with higher MOI (Fig S1D), parasite aggregation also increased, making reliable quantification difficult (Fig S1E). Therefore, we selected a $\mathrm{MOI}$ of 0.5 for 90 min for screening. We tested the robustness of the assay by infecting and scoring 24 replicate wells, either with no RNAi, or transfected with RNAi targeting a control (non-silencing) gene, or targeting genes known to be required for phagocytosis ( $\beta C O P$, Cdc42) [25-29] (Fig S2A-B). These phagocytosis genes clearly and significantly reduced amastigote entry.

We then used this assay to identify factors required for the internalization of $L$. amazonensis into SL2 cells by performing a genome-wide RNAi screen with a library of $\sim 13,000$ dsRNAs covering nearly the entire Drosophila genome (Ambion). The number of intracellular parasites per cell for each RNAi treatment was quantified and the Robust $Z$-score was calculated (the distance of the infection in each well from the median of the entire plate) [30].

The entire library was screened in two completely independent replicates and the Zscores of infection from the duplicate screens are presented in the Figure $1 \mathrm{C}$, where $\mathrm{X}$ and $\mathrm{Y}$-axes display the Z-scores of each replicate, and each dot represents a distinct RNAi treatment (the complete dataset of preliminary hits is also presented in Table S1). We used a cutoff of $-\leq-2.0$ or $\geq 2$ in both replicates $(p<0.001)$ to identify the genes required for infection and genes that restrict infection (referred to as 'down 'and 'up' hits hereafter). Further, those candidates that reduced SL2 cell viability and/or proliferation (green dots in Figure 1C) were removed from further analysis. This analysis revealed 120 candidate genes (118 downs and 2 up hits, red and purple dots in Figure 1C). Independent dsRNAs targeting these candidates, free of predicted off-targets [31], were synthesized, and used in validation assays performed in triplicate. This secondary screen validated 61 down hits but neither of the initial candidate ups (Figure 1D; Table S1).

Amongst these 61 down hits, we next sought to determine which generally impact phagocytosis and which are specifically required for uptake of Leishmania amastigotes. 
To this end, we performed phagocytosis assays with FITC-labeled E. coli and $S$. aureus [32]. We quantified uptake of FITC labeled E. coli and $S$. aureus using flow cytometry [29]. Six candidates reduced the internalization of both $E$. coli and $S$. aureus by $\geq 30 \%$ and were thus classified as general phagocytic factors, while 55 were more specific to Leishmania (Fig. 1E, and Table S1). Three of the Leishmania specific hits have no clear human homologs, leaving 52 down hits for further investigations.

\section{Leishmania uptake factors are highly enriched in protein SUMOylation}

In order to identify host cell biological processes affected by the 52 conserved hits from our genome-wide RNA screen, these candidates were submitted to an overrepresentation test using Panther, with the Drosophila GO network [33]. "Protein SUMOylation" was 99-fold enriched with a P-value of $8 \times 10^{-6}$ (Table 1). Three of the eight genes that make up the protein SUMOylation group were hits - both SUMO E1 subunit genes Aos1 and Uba2, as well as the SUMO E3 ligase, Su(var)2-10 (aka PIAS). Additionally, the single Drosophila SUMO ortholog smt3 reduced infection, but also affected cell proliferation, while the SUMO protease velo (SENP6/7 homolog) affected both Leishmania entry and bacterial phagocytosis (Figure 1D-E marked with pink stripes, and Table S1).

\section{L. amazonensis drastically reduces macrophage SUMOylation}

We next set out to define a role for protein SUMOylation in Leishmania infection of mammalian macrophages. We infected mouse bone marrow derived macrophages (BMDMs) with L. amazonensis amastigotes and harvested whole cell lysates at various time points after infection; total protein SUMOylation was analyzed by immunoblotting for SUMO1 or SUMO2/3 (Figure 2A, B, left). Uninfected macrophages presented numerous SUMO-conjugated bands ranging from $\sim 60$ to $>250 \mathrm{kDa}$. This overall SUMOylation signal visibly decreased $2 \mathrm{~h}$ after L. amazonensis amastigote infection and was nearly undetectable by $48 \mathrm{~h}$ post infection, with either SUMO1 or SUMO2/3 probes. The infection did not change the quantity of free SUMO2/3 bands (Figure 2A, $15 \mathrm{kDa}$ band), free SUMO1 was not detectable. This loss of SUMOylation was not due to total protein degradation or loss, as normal levels of GAPDH and total protein were 
observed by immunoblotting or Coomassie Blue staining, respectively (Figure 2 at bottom of each blot and S3).

The sandfly bite transmits the promastigote form of the parasite to human skin, where they infect phagocytes by receptor mediated phagocytosis [34]. Once internalized, promastigotes differentiate into amastigotes, a differentiation process that typical requires $48 \mathrm{~h}-72 \mathrm{~h}$ [35]. Interestingly, BMDM infection with promastigotes caused a delayed reduction of SUMO1 and SUMO2/3 conjugation, which was observed only after $48 \mathrm{~h}$ of infection (Figure 2A, B, middle). This delayed inhibition of SUMOylation with promastigote infection correlates with the time required for differentiation of promastigote into amastigotes [35].

Phagocytosis of heat-killed amastigotes slightly reduced SUMO1ylation at $24 \mathrm{~h}$ but no differences were observed for SUMO2/3 (Figure 2A-B, right). All together, these data argue that live intracellular amastigotes, but not promastigotes, are potent inhibitors of overall macrophage protein SUMOylation.

Next, we investigated if the intracellular parasites were able to inhibit protein SUMOylation induced by heat shock, a classic inducer of elevated SUMOylation [36]. BMDMs were infected with amastigotes for various times, and then were heat shocked at $43^{\circ} \mathrm{C}$ for $30 \mathrm{~min}$. Similar to observations in the human lymphoblast cell line K-562 [37], heat shock induced the SUMOylation of high molecular weight proteins in uninfected BMDMs (Figure 2C, NI). However, this SUMOylation was strongly inhibited in cells infected with amastigotes for $2 \mathrm{~h}, 8 \mathrm{~h}$ or $24 \mathrm{~h}$ (Figure 2C), indicating that Leishmania parasites actively suppress SUMOylation induced by external stimuli.

To determine if similar regulation of SUMOylation occurs in human systems, THP-1 macrophages [38] were similarly analyzed following amastigote infection. The infection of these human macrophages with amastigotes drastically reduced overall levels of protein SUMO2/3-ylation with little or no change in free SUMO2/3 (Figure 2D). The overall protein SUMO2/3ylation was at undetectable levels at $72 \mathrm{~h}$ post infection. In addition, a THP-1 cell line was generated that constitutively over-expresses HASUMO2, which exhibited increased levels of free SUMO2 as well as SUMOylated proteins in uninfected cells (Figure 2D). While the loss of SUMOylation was delayed in 
this case, parasite infection still reduced it to undetectable levels by 72 hours post infection.

\section{SUMOylation inhibition favors Leishmania infection}

Several pathogens sabotage the SUMOylation machinery to better survive in the vertebrate host [39-41]. We hypothesized that protein SUMOylation attenuates amastigote proliferation, and the inhibition of SUMOylation by Leishmania amastigotes may enhance their survival or replication. To evaluate this hypothesis, THP-1 cells were transduced with lentivirus stably expressing shRNAs specifically targeting SUMO1 or SUMO2. The efficiency of knockdowns was approximately $50 \%$, determined by qRTPCR (Figure 3A). The initial number of parasites per cell was not significantly affected, indicating amastigote entry was unaffected (Figure 3B, 6 h post infection). However, by $72 \mathrm{~h}$ the number of parasites per infected cell was significantly higher in SUMO2-knock down cells compared to the control cells (Fig 3B). SUMO1 knockdown trended in the same direction but did not reach statistical significance. These data demonstrate that $L$. amazonensis replicates more effectively in macrophages with reduced SUMOylation capacity. These findings are consistent with our hypothesis that SUMO-dependent process interfere with amastigote growth and argue that amastigotes restrain macrophage SUMOylation to enhance their intracellular niche and increase parasite growth.

\section{SUMOylation inhibition promotes PV enlargement and drives expression of} ATP6V0D2

Intracellular amastigotes from the L. mexicana group, including L. amazonensis induce the biogenesis of enlarged PVs, which favor their survival and replication. We investigated the hypothesis that reduction of SUMOylation could promote PV biogenesis. Indeed, macrophages with knockdown of SUMO1 or SUMO2 presented larger PVs than control cells (Figure 3C-D), consistent with the hypothesis that disruption of macrophage SUMOylation favors PV maturation and thereby enhances parasite growth.

The mechanisms driving the PV biogenesis are of great interest but still poorly understood. In our previous work we demonstrated that the scavenger receptor CD36 
participates in the biogenesis of L. amazonensis PV while Pessoa et al. (2019) reported that an alternate subunit of the V-ATPase, Atp6v0d2, is involved in PV enlargement and parasite survival by regulating CD36 expression and cholesterol retention [42]. Thus, we investigated if SUMOylation inhibition promotes the expression of ATP6V0D2, CD36 or cholesterol retention.

THP-1 macrophages, control and SUMO-depleted, were infected with amastigotes and expression of ATP6V0D2 and CD36 determined by qRT-PCR. In wild type cells, parasite infection induced expression of ATP6V0D2 3 to 5 -fold at $6 \mathrm{~h}$ to $24 \mathrm{~h}$ pi. Moreover, ATP6VOD2 expression was significantly higher in SUMO1 and SUMO2 knockdown macrophages, including higher induction post infection (Fig. 4A). The expression of $C D 36$ was not consistently elevated by infection in control or knockdown cells, but SUMO1/2 knockdown cells presented generally higher levels (Fig. 4B).

It was previously shown that knockdown of Atp6v0d2 reduces the macrophage overall cholesterol levels and impairs PV biogenesis [42]. In fact, SUMO2 depleted cells, with elevated ATP6V0D2 expression, also presented higher levels two key genes of the cholesterol biosynthesis pathway, HMGCR and SQLE, while SQLE was similarly elevated only in SUMO1 knockdown (Fig 4C-D). Moreover, lysates of SUMO depleted cells contained higher levels of total cholesterols (Figure 4E). Together, these results indicate that SUMOylation inhibition, perhaps through its effect on ATP6VOD2 expression, increases expression of CD36 and some cholesterol biosynthetic genes and intracellular cholesterol levels, favoring PV biogenesis.

\section{Atp6v0d2 expression is sufficient to increase free cholesterol, CD36 expression and promote PV expansion in macrophages}

Similar to human THP-1 macrophages, infection of RAW 264.7 cells with amastigotes promoted the expression of Atp6v0d2 (Fig. 5A). To evaluate if Atp6v0d2 induction is sufficient for the changes observed in SUMO depleted macrophages we generated RAW 264.7 macrophages stably expressing this gene. [Note, THP-1 cells were not used in these experiments because constitutive ATP6VOD2 expression was toxic to THP-1 cells.] Interestingly, ATP6VOD2 expressing RAW 264.7 cells phenocopied many aspects of SUMO depleted THP-1 macrophages, with increased the 
expression of CD36 and significantly higher levels of free cholesterols (Fig. 5B-C). However, ATP6VOD2 overexpression did not induce the expression of cholesterol biosynthetic genes in RAW 264.7 cells (Figure S4), suggesting that higher levels of cholesterol observed in this cell line is linked to increased lipid intake by CD36. Importantly, Atp6v0d2 expression strongly promoted PV biogenesis (Fig. 5D-E). Together, these results show that intracellular amastigotes inhibit SUMOylation to increase ATP6V0D2 expression, which leads to increased cholesterol intake and drives PV enlargement and parasite proliferation, in human and mouse macrophages.

\section{Discussion}

This work began with a genome-wide RNAi screen in Drosophila SL2 cells to identify novel factors involved in L. amazonensis amastigote infection. The screen was designed to identify factors involved in the initial infection steps, when phagocytes recognize and engulf parasites, potentially triggering cellular responses that can affect the downstream fate of infection such as PV biogenesis. Drosophila phagocytes have been successfully used to study several human pathogens, including intracellular bacteria, viruses, and protozoan parasites [26, 43-47]. The conservation of key recognition and signaling pathways involved in host defense and phagocytosis, including Leishmania uptake, was highlighted by the findings from our screen where 52 of 55 genes identified for their specific effects on L. amazonensis infection, are conserved between insects and humans.

We did not find common hits between our results and the published work from Peltan et al. (2012), who screened for L. dononovani amastigote infection factors also in Drosophila S2 cells [26]. This emphasizes the large phylogenetic distance between these two New World Leishmania species. Another explanation for the lack of concordane is that $L$. donovani screen used a dsRNA library with only 1,920 conserved genes compared to our genome wide library targeting 13,071 genes. The library used for $L$. donovani screen did not contain, for example, three SUMOylation factors, AOS1, Su(var)2-10 and UBA2, identified in our screen.

Based on the strong SUMOylation signature in the screen results, we explored the role of SUMOylation in Leishmania infection of mammalian macrophages. Interestingly 
amastigote, but not promastigote, infection quickly and potently inhibited host SUMOylation in both human and mouse macrophages, even inhibiting the induction of SUMO conjugation observed with heat shock. This drastic protein deSUMOylation seems to favor infection because knockdown of SUMOylation factors prior to infection caused higher proliferation of amastigotes within larger macrophages PVs. Together these data suggest $L$. amazonensis amastigotes actively interfere with the host cell SUMO machinery to enhance their own replicative niche.

SUMO regulates numerous cellular functions and activities, and human cells have 3,500 to 6,700 SUMOylated proteins, many of them SUMOylated on multiple residues $[17,48-50]$. Not surprisingly, many pathogens sabotage this post-translational modification to facilitate infection. For example, bacteria Salmonella typhimurium, Listeria monocytogenes, Shigella flexneri and Yersinia pestis actively interfere the SUMOylation machinery during intracellular invasion [18-20, 51]. DeSUMOylation caused by L. monocytogenes inhibits TGF beta signaling, while S. flexneri-induced deSUMOylation increases permeability of the gut, favoring bacterial invasion and induces an exacerbated inflammatory response in mice [20]. Intracellular $S$. typhimurium reduces Rab7 SUMOylation and favors the formation of Salmonellainduced membrane filaments radiating from the Salmonella-containing vacuole which improves bacteria survival [21]. Finally, two protozoan parasites, which also live in parasitophorous vacuoles, Plasmodium berghei and Toxoplasma gondii, reduce protein SUMOylation to inhibit nuclear translocation of immune induced transcription factors, such as SMAD4, thereby reducing cellular responses to infection, resisting apoptosis, and favoring parasite infection [39].

Our data support the hypothesis that amastigotes cause a rapid inhibition of host protein SUMOylation to induce ATP6V0D2 expression and PV enlargement to facilitate survival of intracellular amastigotes. The enlarged PV, typical of parasites from the $L$. mexicana group, is a specialized organelle that protects the intracellular parasites from anti-parasitic host defense such as nitric oxide [52]. PV enlargement is actively driven by amastigotes, which manipulate macrophage vesicle trafficking and lipid metabolism/transport for this purpose; however the mechanisms used by $L$. amazonensis to regulate these processes are largely unknown [53, 54]. Here we 
propose that SUMOylation inhibition is a Leishmania virulence mechanism that increases availability of host cell cholesterol, promotes PV enlargement and favors infection.

One striking finding was that SUMOylation inhibition promotes the expression of ATP6V0D2, a protein previously reported to be involved in PV biogenesis [42]. Atp6v0d2 is best known as a component of vacuolar (V-)ATPases. V-ATPases are multiunit complexes composed by two domains: $\mathrm{V}_{1}$ domain, responsible for ATP hydrolysis and generation of energy for the proton translocation through the integral $\mathrm{V}_{0}$ domain. Atp6v0d subunits are part of the $\mathrm{V}_{0}$ domain and forms a connective stalk between subunits $\mathrm{V}_{0}$ and $\mathrm{V} 1$. While V-ATPases are present in all cells, Atp6v0d2 is expressed in specific tissues (renal, epididymis, osteoclast, dendritic cells, macrophages), while the more common d-subunit, Atp6v0d1 is expressed ubiquitously $[55,56]$. More recently, Atp6v0d2 has been shown to participate in several cellular processes distinct from V-ATPase activity, including fusion of osteoblasts [57], autophagosome-lysosome fusion and inflammasome activation [58], and the degradation of transcription factors [56]. Thus, this alternate VATPase subunit appears to have roles both in vacuolar acidification and in unrelated processes that often involve membrane fusion.

This study links ATP6V0D2 expression to parasite-triggered deSUMOylation. Previous work has linked Atp6v0d2, Cd36 and cholesterol levels [42]. The role of Cd36 in PV biogenesis was previously reported by our group, where the receptor favors fusion of endolysosomes to PV [25]. Since we observed that ATP6VOD2 (over)expression had variable effects on cholesterol biosynthetic genes, in mouse or human macrophages but consistent effects on CD36 expression, we favor the idea that the increased uptake of cholesterol observed in all these models is primarily mediated by elevated CD36 uptake. Interestingly, our previous work showed CD36 localized to PV membrane in contact with parasites, suggesting this lipid transporter may directly delivery cholesterol to PV membrane, lumen and/or to the parasite membrane. Through these modulations of ATP6VOD2 expression and cholesterol levels, amastigotetriggered deSUMOylation has profound effects parasite intracellular growth. 
One unanswered question is how SUMOylation inhibition promotes ATP6V0D2 expression. The role of SUMOylation in the control of gene expression is wellestablished, particularly via the SUMOylation of transcription factors [59]. Although the effect of transcription factor SUMOylation varies, in most cases it reduces transcription of target genes [60]. Interestingly, a publicly available ChIP-seq dataset from mouse dendritic cells shows that a $588 \mathrm{bp}$ DNA region, 32kb upstream of Atp6v0d2, is significantly enriched in SUMO2-conjugated chromatin [61]. Therefore, one possibility is that amastigote-induced deSUMOylation of transcription factor(s), which bind near this element near Atp6v0d2, drive higher expression of Atp6v0d2. Some potential candidates are the transcription factors CTCF, RAD21, Zinc Finger protein 143, c-FOS and PPARy, whose activities are known to be modulated by SUMOylation and are linked in Atp6v0d2 expression [62, 63].

In summary, our Drosophila screen has proven to be effective for the identification of novel host factors involved in Leishmania-host cell interactions. Our results identify a novel $L$. amazonensis virulence strategy, whereby amastigotes drastically reduce host cell protein SUMOylation to increase ATP6V0D2 expression and cholesterol availability, as a strategy to promote their own proliferation. The Leishmania parasitophorous vacuole has clear parallels to the replicative niche of other intracellular bacterial pathogens and it will be interesting to learn, from future studies, if similar processes are involved. Further in-depth studies focused on the mechanistic interactions between protein SUMOylation and parasite growth, as well as on the other hits found in the Drosophila screen may reveal new targets for leishmaniasis treatment. 


\section{Materials and Methods}

\section{Ethics statement}

Our lab conducted all experiments according to the guidelines of the American Association for Laboratory Animal Science and approved by the Institutional Animal Care and Use Committee at the University of Massachusetts Medical School (Docket\#: A-2056-19).

\section{Mice}

C57BL/6 and Balb/c mice were obtained from The Jackson Laboratory. Seven to twelve-week-old mice were maintained under pathogen-free conditions at the University of Massachusetts Medical School animal facilities.

\section{Macrophages}

BMDM: femurs and tibia were dissected from mice and the bone marrow was flushed with PBS using a $30 \mathrm{G}$ needle connected to a $10 \mathrm{ml}$ syringe. The cells were cultivated in RPMI supplemented with 30\% L929 cell-conditioned medium and 20\% FBS in bacteriological petri dishes $\left(4 \times 10^{6}\right.$ cells/plate) at $37^{\circ} \mathrm{C}$ and $5 \% \mathrm{CO}_{2}$ [67]. The cultures were re-fed on day 3 and used on day 7-10. THP-1 monocytes were differentiated to macrophages by stimulation with $100 \mathrm{ng} / \mathrm{mL}$ of phorbol 12-myristate 13-acetate (PMA) in RPMI $10 \%$ FBS for 2 days at $37^{\circ} \mathrm{C}$, washed and cultured for more $24 \mathrm{~h}$ in complete media without PMA, prior use. RAW 264.7 cells were cultivated in DMEM supplemented with $10 \%$ FBS.

\section{Macrophage transduction}

SUMO1 and SUMO2 were knocked down using GIPZ lentiviral particles from GE Dharmacon V3LHS_404077 and V3LHS_412780. ATP6V0D2 (NM_152565.1) was cloned with a C-terminus tag in the lentiviral vector PCX4-PURO and used to generate lentiviral particles and transduce macrophages. Cells were selected with puromycin before use.

\section{Confocal microscopy}

SL2 cells were seeded ( $2 \times 10^{5}$ cells per dish) in $35 \mathrm{~mm}$ dish with glass bottom one day before infection. Cells were infected with amastigotes and at determined times, cell membranes were stained with CellMask deepRed for $10 \mathrm{~min}$, and acidic organelles were stained with Lysotracker Blue DND-22 (Invitrogen). Confocal images were taken 
using a Leica SP8 confocal microscopy using a 63x objective. Images were edited using Fiji software [64].

\section{Leishmania parasites}

GFP-transfected L. amazonensis (MHOM/BR/1973/M2269), and dsRed-transfected (RAT/BA/LV78) [65] were generously donated by Dr. Silvia R. Uliana (ICB-USP, Brazil) and Dr. Kwang-Poo Chang (Rosalind Franklin University of Medicine and Science), respectively. Promastigotes were cultivated in vitro in M199 medium supplemented with $10 \%$ FBS and $30 \mu \mathrm{g} / \mathrm{mL}$ Hygromycin B (GFP transfected) or $5 \mu \mathrm{g} / \mathrm{mL}$ of Tunicamycin (dsRed transfected) at $26^{\circ} \mathrm{C}$.

\section{Production of amastigote forms}

Promastigote forms were differentiated axenically to amastigote by cultivating the parasites with 199 media supplemented with $0.25 \%$ glucose, $0.5 \%$ trypticase, $40 \mathrm{mM}$ sodium succinate ( $\mathrm{pH} 5.4$ ) and $20 \%$ FBS, at $1 \times 10^{\wedge} 7$ cells $/ \mathrm{mL}$ at $34^{\circ} \mathrm{C}$ for 3 days. Axenic amastigotes were then used to infect Balb/c or C57BL/6 BMDM cultivated in $175 \mathrm{~cm}^{2} \mathrm{~T}$ flasks ( $3 \times 10^{7}$ cells/flask) at a multiplicity of infection of 3 parasites/BMDM. At least 4 days after infection cells were harvested from T-flasks using a cells scraper and disrupted in PBS at $4^{\circ} \mathrm{C}$ using a glass dounce tissue grinder with teflon rod. Parasites were purified by two-step centrifugations, one at $210 \mathrm{~g}$ for $8 \mathrm{~min}$ to remove intact BMDMs and cell debris, and then supernatant was centrifuged at $675 \mathrm{~g}$ for 12 min to precipitate amastigotes.

\section{HTS screening}

For genome wide screen a library of 13,071 dsRNA against $D$. melanogaster genes (Ambion) distributed in 32 384-well plates with clear bottom (Costar) was utilized. SL2 cells were maintained in complete media (Schneider media (Gibco), 10\% fetal bovine serum (Optima, Atlanta Biologicals), $100 \mathrm{iU}$ of penicillin and streptomwycin) at $27^{\circ} \mathrm{C}$. After changing to serum-free media, 20,000 cells in ten $\mu \mathrm{L}$ of was added to each well, which contained $250 \mathrm{ng}$ dsRNA, and incubated for $1 \mathrm{~h}$, then $20 \mu \mathrm{l}$ of complete media was added per well and incubated for $72 \mathrm{~h}$ for knockdown. GFP expressing amastigotes harvested from BMDMs (104 in $5 \mu$ l of Schneider media per well) were added to each well and the plates centrifuged at $300 \times \mathrm{G}$ for $5 \mathrm{~min}$ and kept at $27 \mathrm{C}$ for $90 \mathrm{~min}$. The media was carefully removed from wells and cells were fixed with $20 \mu$ of $3.2 \%$ formaldehyde (EM grade, EMS) for $30 \mathrm{~min}$ at room temperature and not permeabilized. 
The cultures were washed once with PBS and blocked with $1 \%$ bovine serum albumin (BSA) for $1 \mathrm{~h}$ at $37^{\circ} \mathrm{C}$. Extracellular parasites were immunostained with serum of an infected Balb/c diluted 1:4000 in PBS with 1\% BSA, and a secondary goat anti-mouse conjugated to Alexafluor 594 1:1000 (Invitrogen). The DNA of cells were counterstained using $0.1 \mu \mathrm{g} / \mathrm{ml}$ Hoechst 33342 added to the secondary antibody solution, the general scheme for the method is shown in Figure S2. Three fields per well, close to the center of wells, were imaged using a 20x objective using an ImageXpress Micro automated microscope and analyzed using MetaXpress software (Molecular Devices). Number of SL2 cells (Hoechst 33342 staining, total parasites (GFP positive), and extracellular parasites (GFP and AlexaFluor 594 positive), intracellular parasites (GFP positive and AlexaFluor 594 negative) were scored and averaged for the three images of each well. The infection rate (number of intracellular parasites per SL2 cells) was log-transformed, and the median and interquartile range were used to calculate a Z-score: (log10(\%infection)-log10(median))/(IQR*0.74) for each plate [30]. The entire screen was performed in duplicate and wells with Robust Z-scores of infection $\geq 1.5$ or $\leq-2.0$ in both replicates were considered 'hits'. Knockdowns that reduced the SL2 numbers by -2 Z-scores received lower priority to further studies.

\section{dsRNA Synthesis and secondary screen}

Primer sequences to generate dsRNA sequences with no off-targets were obtained from DRSC/TRiP Functional Genomics Resources (www.flyrnai.org) or manually designed using SnapDragon (https://www.flyrnai.org/cgi-bin/RNAi_find_primers.pl) (Supplemental Table 2). After PCR using genomic DNA from flies, dsRNA was synthesized using MEGAscript T7 transcription kit (Invitrogen) following manufacturer recommendations.

The secondary screen plates were arrayed with dsRNA (250 ng) using on-site synthesized dsRNA free of off targets. Genes were considered hits when two of the triplicates Robust Z-scores in infection was $\geq 1.5$ or $\leq 1.5$ using dsRNA against 180 wells treated with dsRNA against $\beta$-galactosidase.

\section{Phagocytosis of E. coli and S. aureus}

SL2 cells were soaked with $1.25 \mu \mathrm{g}$ of dsRNAs in 96 well plate for 3 days prior to phagocytosis assays. Fluorescein-labeled E. coli (K-12 strain), and Staphylococcus 
aureus (Wood strain, without protein A) (Molecular Probes), were washed 3 times with PBS by centrifugation and sonicated for 3 times at $50 \mathrm{KHz}$ for $20 \mathrm{~s}$. Twenty micrograms of bacterial particles were added to each well at $4^{\circ} \mathrm{C}$, the plates were centrifuged $300 \mathrm{xg}$ for $3 \mathrm{~min}$ at $4^{\circ} \mathrm{C}$, and incubated in a $27^{\circ} \mathrm{C}$ water bath for 20 or $40 \mathrm{~min}$ (for E. coli and $\mathrm{S}$. aureus, respectively). On ice bath, cells and bacteria were removed from plates by pipetting 300 ul of a $0.1 \%$ Trypan Blue solution in PBS pH 5.4 to quench the fluorescein from extracellular bacteria. The bacteria inside live cells was quantified by flow cytometry [29].

\section{Bioinformatic analysis}

The clustering and enrichment analysis of the screen hits was performed in PANTHER Classification System [66].

\section{Western Blot}

Macrophages were lysed in standard lysis buffer containing $10 \%$ glycerol, $1 \%$ Triton $\mathrm{X}$ 100, $20 \mathrm{mM}$ Tris, $150 \mathrm{mM} \mathrm{NaCl}, 24 \mathrm{mM} \beta$-glycerol phosphate, $2 \mathrm{mM}$ EDTA, $1 \mathrm{mM}$ 1,4 dithiothreitol (DTT), $1 \mathrm{mMsodium}$ orthovanadate, $5 \mathrm{mM} \mathrm{N}$-ethylmaleimide, $0.5 \mathrm{U} / \mu \mathrm{l}$ Benzonase, $1 \times$ protease inhibitor mixture (Halt, Thermofisher). After protein quantification by Bradford protein assay (Bio-Rad), Laemmli sample buffer $4 x$ was added to samples and heated for $3 \mathrm{~min}$ at $95^{\circ} \mathrm{C}$. For whole cell lysate analysis, $50 \mu \mathrm{g}$ of total protein was separated by SDS-PAGE using $8 \%$ or $4-20 \%$ polyacrylamide gels, and transferred to PVDF membranes at $350 \mathrm{~mA}$ using BioRad Mini Protean and Transblot system (Bio-Rad). Membranes were probed with antibodies against SUMO1 or SUMO2/3 (Cell Signaling $\mathrm{C} 9 \mathrm{H} 1$ and $18 \mathrm{H} 8$ ) using manufacturer conditions. For loading controls, the same blots were probed with anti-actin or anti-GAPDH, and then stained with Coomassie blue (sc8432, Santa Cruz, G9295 Millipore Sigma).

\section{Production of retroviral vectors and transduction of immortalized mouse macrophages}

The full length mouse SUMO2 fused to HA (N-terminus) from a plasmid (Addgene 48967) [67] were inserted in the retroviral transfer plasmid pCX4 Puro. For the viral production, HEK 293T cells were transfected with the pCX4 HA-SUMO2, MLV gag-pol, and VSVG using GeneJuice transfection reagent (EMD Millipore) following the manufacturer's recommendations. Media was replaced at $24 \mathrm{~h}$ and collected $48 \mathrm{~h}$ after 
transfection, filtered through a $0.45 \mu \mathrm{m}$ pore filter and stored at $-80{ }^{\circ} \mathrm{C}$ until use. Virus for shRNA transduction were generated using shRNA library (GIPZ, Dharmacon): SUMO2 (V3LHS_412780), SUMO1- (V3LHS_404077), SAE1 V3LHS_304633, UBE2I (V3LHS_376933). THP-1 cells were cultured in the presence of virus particles and 8 $\mu \mathrm{g} / \mathrm{ml}$ Polybrene for $24 \mathrm{~h}$, and then the transduced cells were selected with $3 \mu \mathrm{g} / \mathrm{ml}$ of puromycin for 4 days. Cells were frozen and when the thawed aliquots were subcultured for up to 3 weeks.

\section{ATP6V0D2 Plasmid}

Human ATP6V0D2-FLAG NM_152565.1 was obtained from Sino Biological (HG23801CF) and cloned in PCX4-Puro lentiviral plasmid.

\section{Macrophage infection and PV area measurement}

THP-1 macrophages were infected with amastigotes for $2 \mathrm{~h}$ and the coverslips were washed 3 times with RPMI media and transferred to a new plate. One day after infection the cultures were fixed with $4 \%$ paraformaldehyde for $15 \mathrm{~min}$ and mounted over a glass slide for microscopic imaging in a fluorescence microscope. The area of PV was manually measured using an elliptical tool in FIJI.

\section{RNA and qRT-PCR}

Total RNA from macrophages were isolated using the TRIzol reagent (Invitrogen) following the manufacturer's recommendations. cDNA was synthesized using iScript gDNA Clear cDNA synthesis kit (BioRad) and quantitative PCR analysis was performed using SYBR Green (BioRad). The specificity of amplification was assessed for each sample by melting curve analysis and relative quantification was performed using a standard curve with dilutions of a standard.

Oligonucleotides used in qPCR reactions: 


$\begin{array}{ll}\text { Gene } & \text { Forward } \\ \text { SUM01 } & \text { GGTCTGGACCAAAAGAAGAGGA } \\ \text { Sum02 } & \text { TGGCATGCTCATTCAGCTCT } \\ \text { Sumo1 } & \text { ATTGGACAGGATAGCAGTGAGA } \\ \text { Sumo2 } & \text { AAGGAAGGAGTCAAGACTGAGAA } \\ \text { ATP6VOD2 } & \text { AAGACCGAGAGACCCTCTATCC } \\ \text { Atp6v0d2 } & \text { CAGAGCTGTACTTCAATGTGGAC } \\ \text { CD36 } & \text { GGCTGTGACCGGAACTGTG } \\ \text { Cd36 } & \text { GGCTAAATGAGACTGGGACCATTG } \\ \text { HMGCR } & \text { TGATTGACCTTTCCAGAGCAAG } \\ \text { Hmgcr } & \text { AGCTTGCCCGAATTGTATGTG } \\ \text { SQLE } & \text { TGACAATTCTCATCTGAGGTCCA } \\ \text { Sqle } & \text { ATAAGAAATGCGGGGATGTCAC } \\ \text { RPL37A } & \text { ATTGAAATCAGCCAGCACGC } \\ \text { Hrpt1 } & \text { GAGGAGTCCTGTTGATGTTGCCAG }\end{array}$

\section{Cholesterol measurement}

Macrophages were seeded in 96 well plates (5×10^4 cells per well) and one day later, free and total cholesterol concentration in lysates was determined using Cholesterol/Cholesterol Ester-Glo Assay (Promega) following manufacturer recommendations. Cholesterol concentrations were normalized by the protein concentration determined by Bio-Rad Protein Assay Dye (Bio-Rad).

\section{Statistical analysis}

The statistical analyses were performed using GraphPad Prism version 6.00 for Mac OS X, GraphPad Software, La Jolla California USA, www.graphpad.com. The tests and the criteria used for each comparison are reported in the Figure legends. 


\section{Bibliography}

1. Guy, R.A. and M. Belosevic, Comparison of receptors required for entry of Leishmania major amastigotes into macrophages. Infect Immun, 1993. 61(4): p. 1553-8.

2. Peters, C., et al., The Role of Macrophage Receptors in Adhesion and Uptake of Leishmania-Mexicana Amastigotes. Journal of Cell Science, 1995. 108: p. 3715-3724.

3. Love, D.C., M. Mentink Kane, and D.M. Mosser, Leishmania amazonensis: the phagocytosis of amastigotes by macrophages. Exp Parasitol, 1998. 88(3): p. 161-71.

4. Wanderley, J.L., et al., Cooperation between apoptotic and viable metacyclics enhances the pathogenesis of Leishmaniasis. PLoS One, 2009. 4(5): p. e5733.

5. Wanderley, J.L., et al., Phosphatidylserine exposure on the surface of Leishmania amazonensis amastigotes modulates in vivo infection and dendritic cell function. Parasite Immunol, 2012. 35(3-4): p. 109-19.

6. de Araujo Soares, R.M., et al., Leishmania (Leishmania) amazonensis: differential expression of proteinases and cell-surface polypeptides in avirulent and virulent promastigotes. Exp Parasitol, 2003. 104(3-4): p. 104-12.

7. Cameron, P., et al., Inhibition of lipopolysaccharide-induced macrophage IL-12 production by Leishmania mexicana amastigotes: the role of cysteine peptidases and the NF-kappaB signaling pathway. J Immunol, 2004. 173(5): p. 3297-304.

8. Mottram, J.C., G.H. Coombs, and J. Alexander, Cysteine peptidases as virulence factors of Leishmania. Curr Opin Microbiol, 2004. 7(4): p. 375-81.

9. Olivier, M., D.J. Gregory, and G. Forget, Subversion mechanisms by which Leishmania parasites can escape the host immune response: a signaling point of view. Clin Microbiol Rev, 2005. 18(2): p. 293-305.

10. Halle, M., et al., The Leishmania surface protease GP63 cleaves multiple intracellular proteins and actively participates in p38 mitogen-activated protein kinase inactivation. $\mathrm{J}$ Biol Chem, 2009. 284(11): p. 6893-908.

11. Contreras, I., et al., Leishmania-induced inactivation of the macrophage transcription factor AP-1 is mediated by the parasite metalloprotease GP63. PLoS Pathog, 2010. 6(10): p. e1001148.

12. Isnard, A., M.T. Shio, and M. Olivier, Impact of Leishmania metalloprotease GP63 on macrophage signaling. Front Cell Infect Microbiol, 2012. 2: p. 72.

13. Kima, P.E., Leishmania molecules that mediate intracellular pathogenesis. Microbes Infect, 2014. 16(9): p. 721-6.

14. Alvar, J., et al., Leishmaniasis worldwide and global estimates of its incidence. PLoS One, 2012. 7(5): p. e35671.

15. Flotho, A. and F. Melchior, Sumoylation: a regulatory protein modification in health and disease. Annu Rev Biochem, 2013. 82: p. 357-85.

16. Gill, G., SUMO and ubiquitin in the nucleus: different functions, similar mechanisms? Genes Dev, 2004. 18(17): p. 2046-59.

17. Hendriks, I.A., et al., Site-specific mapping of the human SUMO proteome reveals comodification with phosphorylation. Nat Struct Mol Biol, 2017. 24(3): p. 325-336.

18. Orth, K., et al., Disruption of signaling by Yersinia effector YopJ, a ubiquitin-like protein protease. Science, 2000. 290(5496): p. 1594-7.

19. Ribet, D., et al., Listeria monocytogenes impairs SUMOylation for efficient infection. Nature, 2010. 464(7292): p. 1192-5.

20. Fritah, S., et al., Sumoylation controls host anti-bacterial response to the gut invasive pathogen Shigella flexneri. EMBO Rep, 2014. 15(9): p. 965-72.

21. Mohapatra, G., et al., A SUMOylation-dependent switch of RAB7 governs intracellular life and pathogenesis of Salmonella Typhimurium. J Cell Sci, 2019. 132(1). 
22. Verma, S., et al., Salmonella Engages Host MicroRNAs To Modulate SUMOylation: a New Arsenal for Intracellular Survival. Mol Cell Biol, 2015. 35(17): p. 2932-46.

23. Lowrey, A.J., W. Cramblet, and G.L. Bentz, Viral manipulation of the cellular sumoylation machinery. Cell Commun Signal, 2017. 15(1): p. 27.

24. Gold, K.S. and K. Bruckner, Macrophages and cellular immunity in Drosophila melanogaster. Semin Immunol, 2015. 27(6): p. 357-68.

25. Okuda, K., et al., Leishmania amazonensis Engages CD36 to Drive Parasitophorous Vacuole Maturation. PLoS Pathog, 2016. 12(6): p. e1005669.

26. Peltan, A., et al., Identification of Drosophila gene products required for phagocytosis of Leishmania donovani. PLoS One, 2012. 7(12): p. e51831.

27. Morehead, J., I. Coppens, and N.W. Andrews, Opsonization Modulates Rac-1 Activation during Cell Entry by Leishmania amazonensis. Infection and Immunity, 2002. 70(8): p. 4571-4580.

28. Hackam, D.J., et al., Indirect role for COPI in the completion of FCgamma receptormediated phagocytosis. J Biol Chem, 2001. 276(21): p. 18200-8.

29. Ramet, M., et al., Functional genomic analysis of phagocytosis and identification of a Drosophila receptor for E. coli. Nature, 2002. 416(6881): p. 644-8.

30. Zhang, X.D., et al., Robust statistical methods for hit selection in RNA interference highthroughput screening experiments. Pharmacogenomics, 2006. 7(3): p. 299-309.

31. Hu, Y., et al., FlyRNAi.org-the database of the Drosophila RNAi screening center and transgenic RNAi project: 2017 update. Nucleic Acids Res, 2017. 45(D1): p. D672-D678.

32. Wan, C.P., C.S. Park, and B.H. Lau, A rapid and simple microfluorometric phagocytosis assay. J Immunol Methods, 1993. 162(1): p. 1-7.

33. Mi, H., et al., PANTHER version 14: more genomes, a new PANTHER GO-slim and improvements in enrichment analysis tools. Nucleic Acids Res, 2019. 47(D1): p. D419D426.

34. Ueno, N. and M.E. Wilson, Receptor-mediated phagocytosis of Leishmania: implications for intracellular survival. Trends Parasitol, 2012. 28(8): p. 335-44.

35. Courret, N., et al., Kinetics of the intracellular differentiation of Leishmania amazonensis and internalization of host MHC molecules by the intermediate parasite stages. Parasitology, 2001. 122(Pt 3): p. 263-79.

36. Seifert, A., et al., Proteotoxic stress reprograms the chromatin landscape of SUMO modification. Sci Signal, 2015. 8(384): p. rs7.

37. Niskanen, E.A., et al., Global SUMOylation on active chromatin is an acute heat stress response restricting transcription. Genome Biol, 2015. 16: p. 153.

38. Mehta, S.R., et al., Flow cytometric screening for anti-leishmanials in a human macrophage cell line. Exp Parasitol, 2010. 126(4): p. 617-20.

39. Maruthi, M., et al., Modulation of host cell SUMOylation facilitates efficient development of Plasmodium berghei and Toxoplasma gondii. Cell Microbiol, 2017. 19(7).

40. Wilson, V.G., Viral Interplay with the Host Sumoylation System. Adv Exp Med Biol, 2017. 963: p. 359-388.

41. Ribet, D. and P. Cossart, Ubiquitin, SUMO, and NEDD8: Key Targets of Bacterial Pathogens. Trends Cell Biol, 2018. 28(11): p. 926-940.

42. Pessoa, C.C., et al., ATP6V0d2 controls Leishmania parasitophorous vacuole biogenesis via cholesterol homeostasis. PLoS Pathog, 2019. 15(6): p. e1007834.

43. Cheng, L.W. and D.A. Portnoy, Drosophila S2 cells: an alternative infection model for Listeria monocytogenes. Cell Microbiol, 2003. 5(12): p. 875-85.

44. Agaisse, H., et al., Genome-wide RNAi screen for host factors required for intracellular bacterial infection. Science, 2005. 309(5738): p. 1248-51.

45. Cherry, S., et al., Genome-wide RNAi screen reveals a specific sensitivity of IREScontaining RNA viruses to host translation inhibition. Genes Dev, 2005. 19(4): p. 445-52. 
46. Philips, J.A., E.J. Rubin, and N. Perrimon, Drosophila RNAi screen reveals CD36 family member required for mycobacterial infection. Science, 2005. 309(5738): p. 1251-3.

47. Cherry, S., et al., COPI activity coupled with fatty acid biosynthesis is required for viral replication. PLoS Pathog, 2006. 2(10): p. e102.

48. Mattoscio, D., C.V. Segre, and S. Chiocca, Viral manipulation of cellular protein conjugation pathways: The SUMO lesson. World J Virol, 2013. 2(2): p. 79-90.

49. Wimmer, P. and S. Schreiner, Viral Mimicry to Usurp Ubiquitin and SUMO Host Pathways. Viruses, 2015. 7(9): p. 4854-72.

50. Lamoliatte, F., et al., Uncovering the SUMOylation and ubiquitylation crosstalk in human cells using sequential peptide immunopurification. Nat Commun, 2017. 8: p. 14109.

51. Sidik, S.M., et al., Shigella infection interferes with SUMOylation and increases PML-NB number. PLoS One, 2015. 10(4): p. e0122585.

52. Wilson, J., et al., Control of parasitophorous vacuole expansion by LYST/Beige restricts the intracellular growth of Leishmania amazonensis. PLoS Pathog, 2008. 4(10): p. e1000179.

53. Young, J. and P.E. Kima, The Leishmania Parasitophorous Vacuole Membrane at the Parasite-Host Interface. Yale J Biol Med, 2019. 92(3): p. 511-521.

54. Courret, N., et al., Biogenesis of Leishmania-harbouring parasitophorous vacuoles following phagocytosis of the metacyclic promastigote or amastigote stages of the parasites. J Cell Sci, 2002. 115(Pt 11): p. 2303-16.

55. Toei, M., R. Saum, and M. Forgac, Regulation and isoform function of the V-ATPases. Biochemistry, 2010. 49(23): p. 4715-23.

56. Liu, N., et al., Lactate inhibits ATP6V0d2 expression in tumor-associated macrophages to promote HIF-2alpha-mediated tumor progression. J Clin Invest, 2019. 129(2): p. 631646.

57. Kim, K., et al., NFATc1 induces osteoclast fusion via up-regulation of Atp6v0d2 and the dendritic cell-specific transmembrane protein (DC-STAMP). Mol Endocrinol, 2008. 22(1): p. 176-85.

58. Xia, Y., et al., The macrophage-specific V-ATPase subunit ATP6V0D2 restricts inflammasome activation and bacterial infection by facilitating autophagosome-lysosome fusion. Autophagy, 2019. 15(6): p. 960-975.

59. Boulanger, M., et al., SUMO and Transcriptional Regulation: The Lessons of LargeScale Proteomic, Modifomic and Genomic Studies. Molecules, 2021. 26(4).

60. Rosonina, E., et al., Regulation of transcription factors by sumoylation. Transcription, 2017. 8(4): p. 220-231.

61. Decque, A., et al., Sumoylation coordinates the repression of inflammatory and anti-viral gene-expression programs during innate sensing. Nat Immunol, 2016. 17(2): p. 140-9.

62. Stelzer, G., et al., The GeneCards Suite: From Gene Data Mining to Disease Genome Sequence Analyses. Curr Protoc Bioinformatics, 2016. 54: p. 130 1-1 3033.

63. Jennewein, C., et al., Sumoylation of peroxisome proliferator-activated receptor gamma by apoptotic cells prevents lipopolysaccharide-induced NCoR removal from kappaB binding sites mediating transrepression of proinflammatory cytokines. J Immunol, 2008. 181(8): p. 5646-52.

64. Schindelin, J., et al., Fiji: an open-source platform for biological-image analysis. Nat Methods, 2012. 9(7): p. 676-82.

65. Dutta, S., et al., Photodynamic sensitization of Leishmania amazonensis in both extracellular and intracellular stages with aluminum phthalocyanine chloride for photolysis in vitro. Antimicrob Agents Chemother, 2005. 49(11): p. 4474-84.

66. Thomas, P.D., et al., PANTHER: a library of protein families and subfamilies indexed by function. Genome Res, 2003. 13(9): p. 2129-41. 
bioRxiv preprint doi: https://doi.org/10.1101/2021.11.03.467107; this version posted November 4, 2021. The copyright holder for this preprint (which was not certified by peer review) is the author/funder, who has granted bioRxiv a license to display the preprint in perpetuity. It is made available under aCC-BY-NC-ND 4.0 International license.

67. Bekes, M., et al., The dynamics and mechanism of SUMO chain deconjugation by SUMO-specific proteases. J Biol Chem, 2011. 286(12): p. 10238-47. 

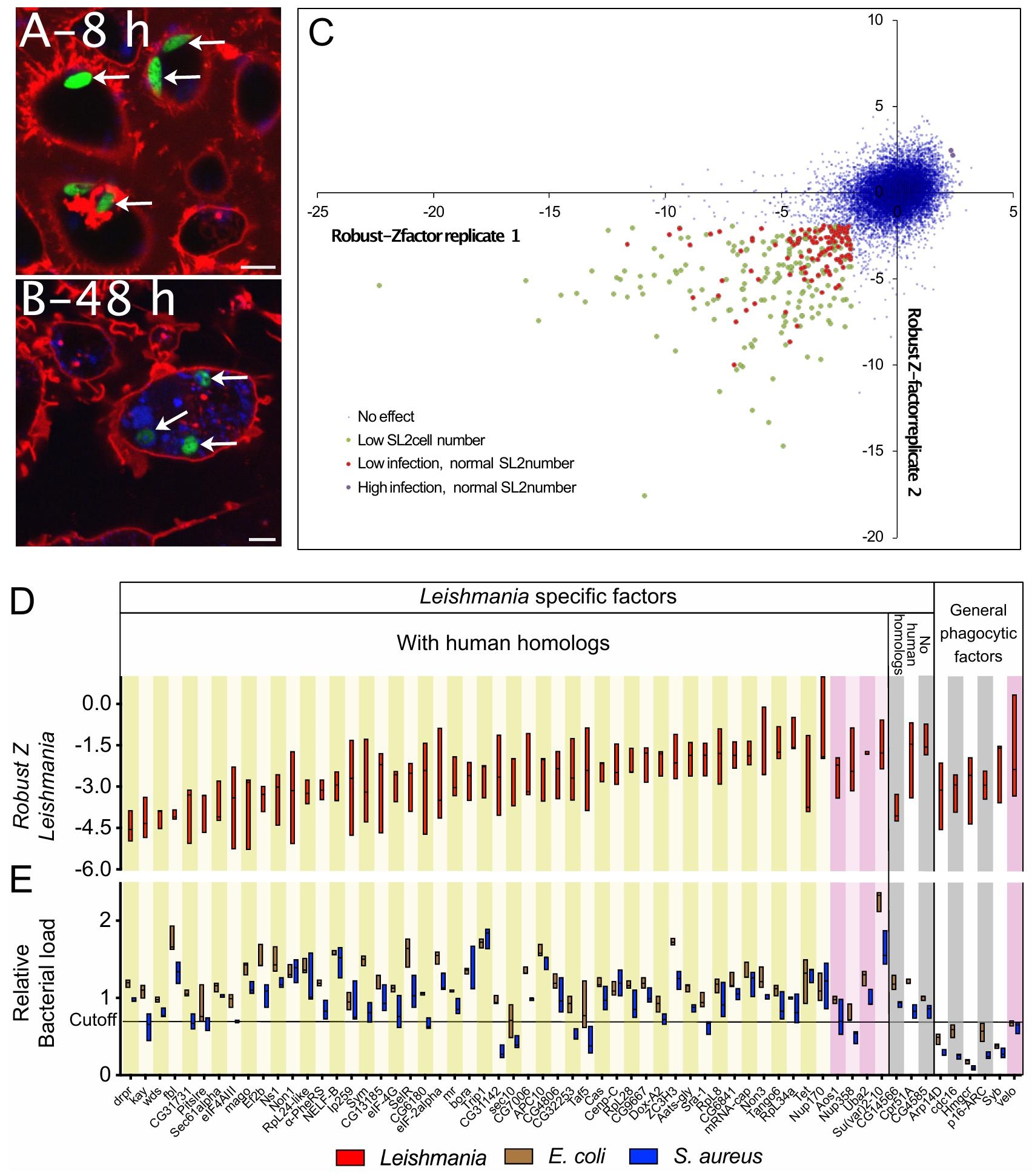
bioRxiv preprint doi: https://doi.org/10.1101/2021.11.03 467107; this version posted November 4, 2021. The copyright holder for this preprint (which was not certified by peer review) is the author/funder, who has granted bioRxiv a license to display the preprint in perpetuity. It is made available under aCC-BY-NC-ND 4.0 International license.
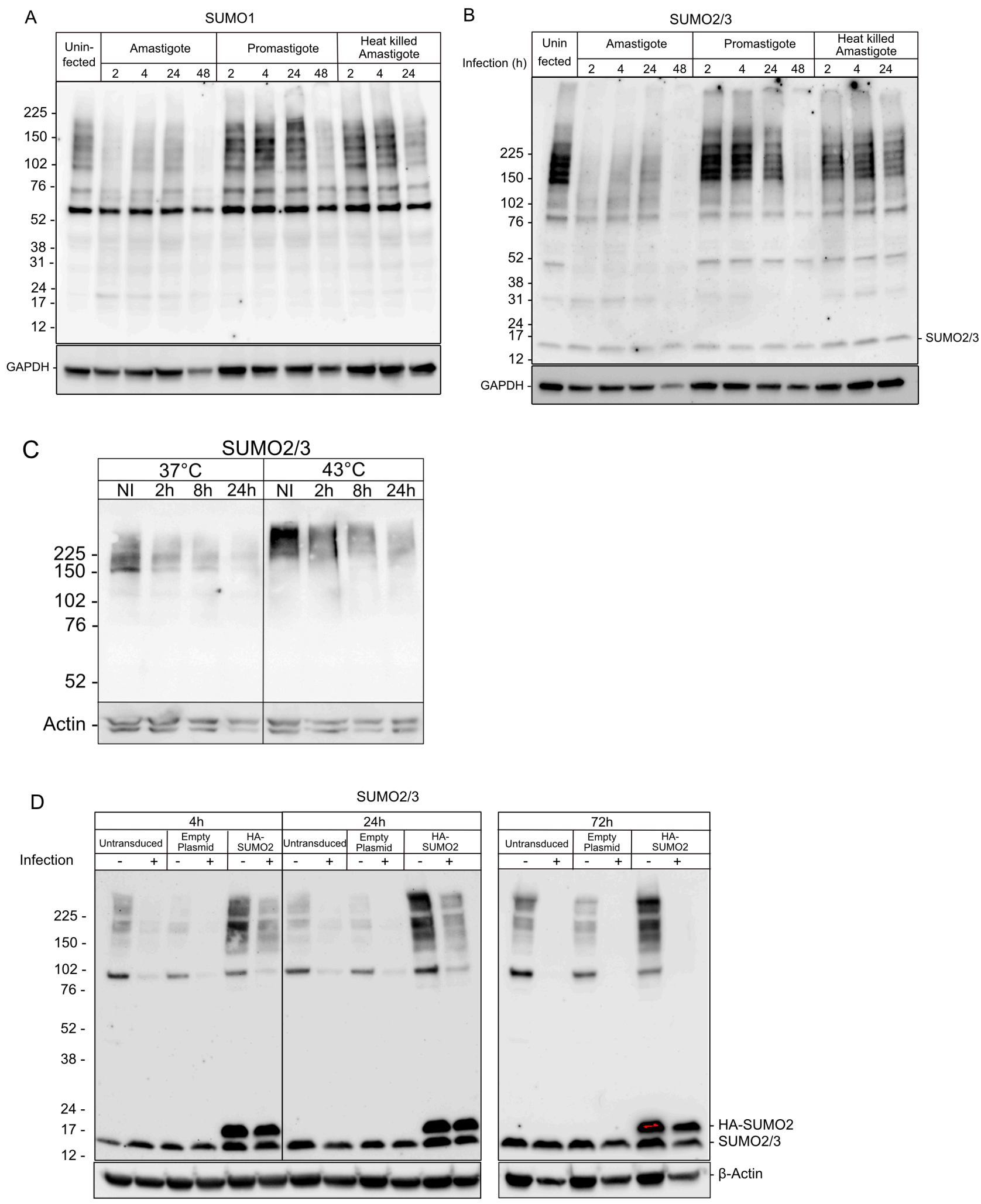
A $\square$ SUMO1 $\square$ SUMO2
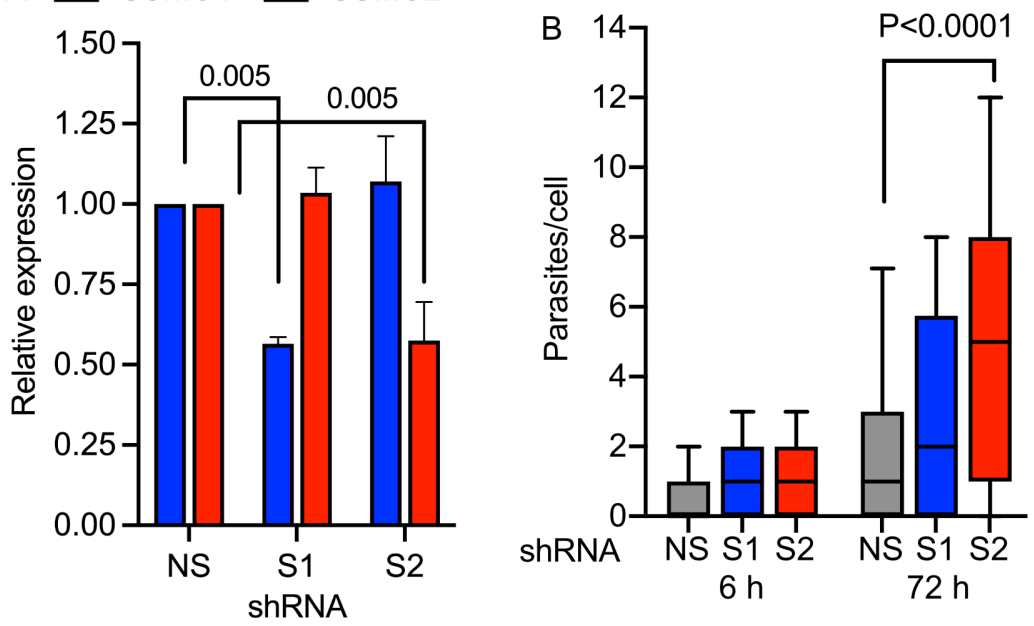

C

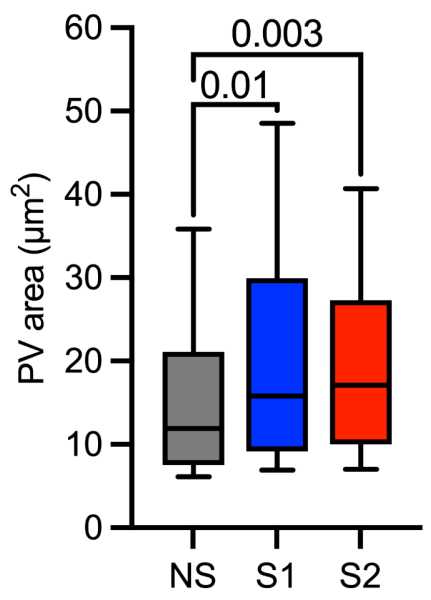

D
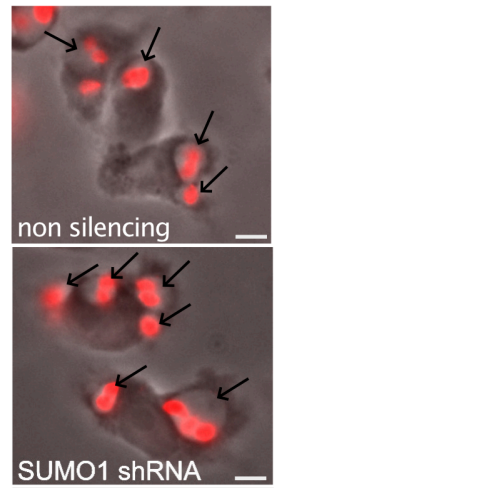


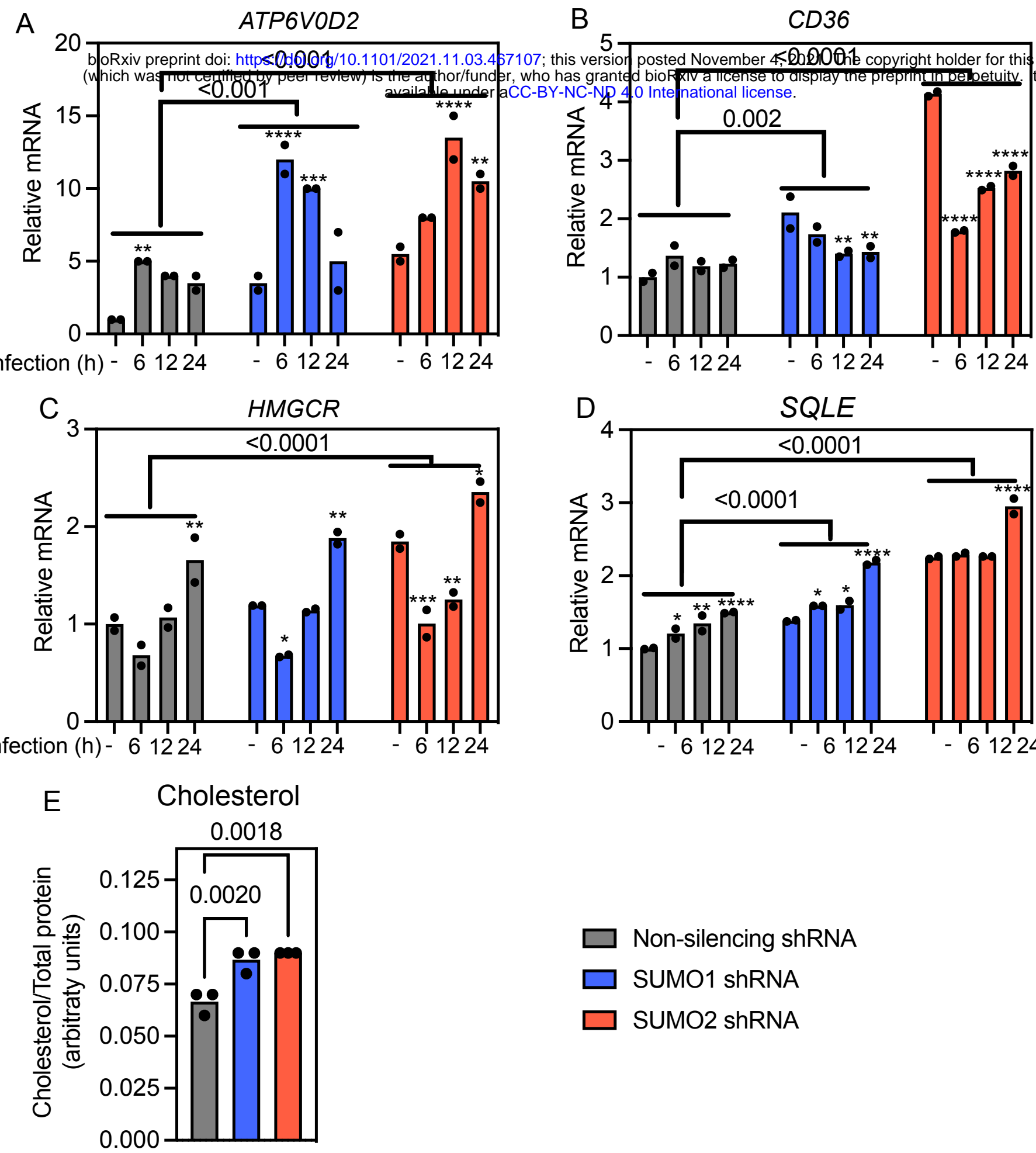



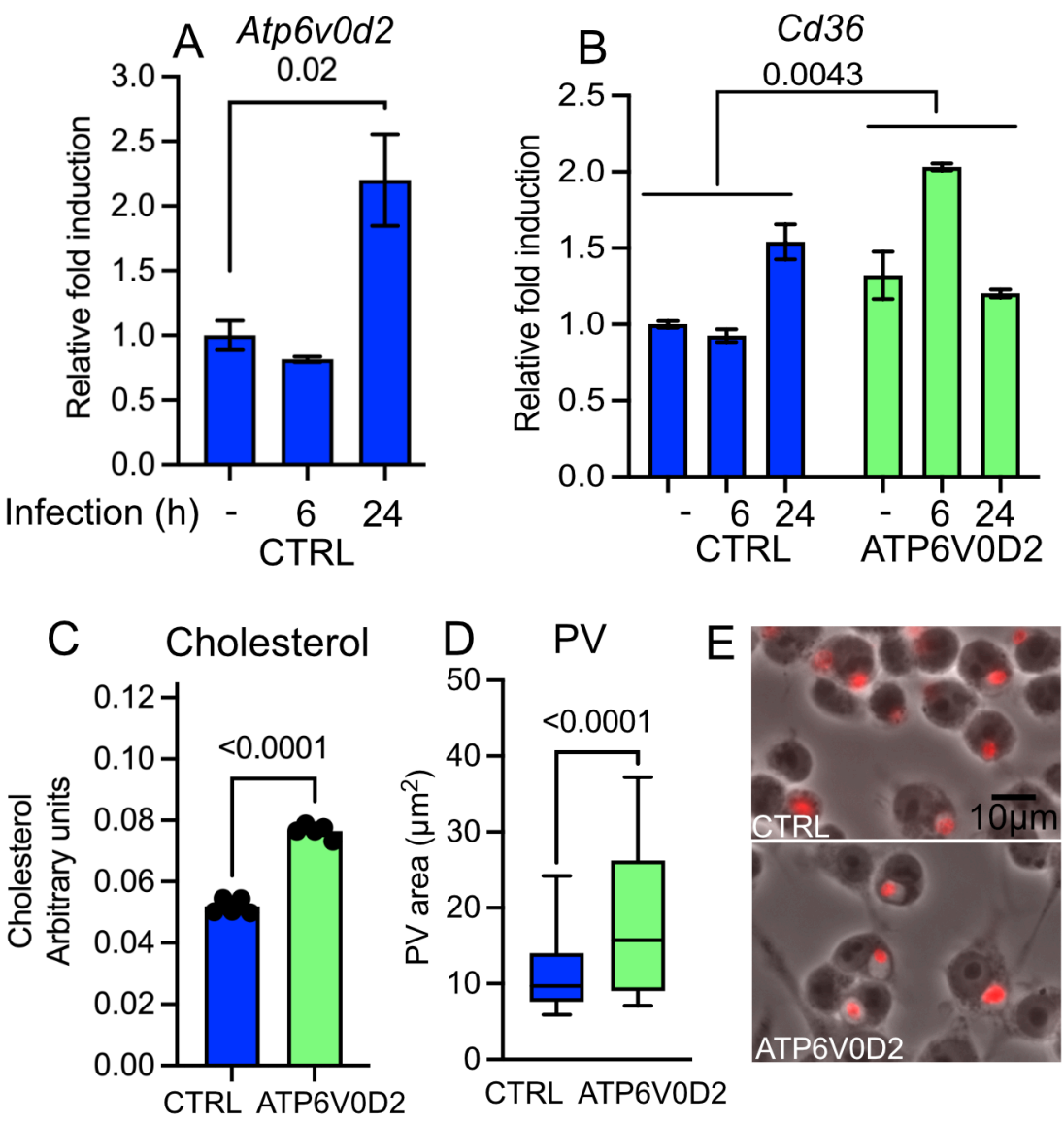
146

6

10.88

2.15E-05

PitsIre, Su(var)2-10, Sec61alpha, Aos1, kay, Uba2

GlyRS, RpL28, EF2, elF2alpha, RpL8, elF3i, RpL24, RpL34, elF4G1, alpha-PheRs Tet, GlyRS, Taf5, mago, Non3, CG7483, mRNAcap, CG13185, CG6841, CG9667, Sym, alphaPheRS 